\title{
Engineering Wavelength Conversion Span in Cascaded Broadband Cherenkov Radiation
}

Wang, Shaofei; Hu, Jungao; Guo, Hairun; Zeng, Xianglong

Published in:

(CLEO EUROPE/IQEC) European Conference on Lasers and Electro-Optics and International Quantum Electronics Conference

Link to article, DOI:

10.1109/CLEOE-IQEC.2013.6801361

Publication date:

2013

Link back to DTU Orbit

Citation (APA):

Wang, S., Hu, J., Guo, H., \& Zeng, X. (2013). Engineering Wavelength Conversion Span in Cascaded Broadband Cherenkov Radiation. In (CLEO EUROPE/IQEC) European Conference on Lasers and ElectroOptics and International Quantum Electronics Conference IEEE. https://doi.org/10.1109/CLEOEIQEC.2013.6801361

\section{General rights}

Copyright and moral rights for the publications made accessible in the public portal are retained by the authors and/or other copyright owners and it is a condition of accessing publications that users recognise and abide by the legal requirements associated with these rights.

- Users may download and print one copy of any publication from the public portal for the purpose of private study or research.

- You may not further distribute the material or use it for any profit-making activity or commercial gain

- You may freely distribute the URL identifying the publication in the public portal 


\title{
Engineering Wavelength Conversion Span in Cascaded Broadband Cherenkov Radiation
}

\author{
Shaofei Wang, ${ }^{1}$ Jungao Hu, ${ }^{1}$ Hairun Guo, ${ }^{2}$ Xianglong Zeng, ${ }^{1,2}$ \\ 1. Key Laboratory of Special Fiber Optics and Optical Access Networks, Shanghai University, Shanghai 200072, China \\ 2. DTU Fotonik, Technical University of Denmark, DK-2800 Kgs. Lyngby, Denmark
}

Optical Cherenkov radiation (OCR) is referred to as dispersive wave (DW) generation or non-solitonic radiation originating from soliton propagation perturbed by high order dispersion (HOD). The OCR becomes of particular importance for octave-spanning spectral broadening and blue-shifted supercontinuum generation (SCG) [1]. When the OCR generation lies in the anomalous GVD region and tends to be a soliton state, the phase-matching (PM) condition between linear DWs and the soliton could become a soliton PM condition: $\beta_{s}\left(\omega_{s}\right)=\beta_{s}\left(\omega_{r}\right)$, provided that $v_{g}\left(\omega_{s}\right)=v_{g}\left(\omega_{r}\right) . \beta_{s}\left(\omega_{k}\right) \equiv \beta\left(\omega_{k}\right)+\left(\omega-\omega_{k}\right) / v_{g}\left(\omega_{k}\right)+q_{k}$ reflects the nondispersive nature of the soliton at the center frequency $\omega_{k}$ and $v_{g}\left(\omega_{k}\right)$ is the group velocity. $k=r, s$ represents the radiation or soliton wave. A sharp spectral switching of the soliton due to the soliton spectral tunneling (SST) effect in the photonic crystal fibers (PCFs) can occur across a normal group velocity dispersion (GVD) region, which is sandwiched in the anomalous GVD regions [2]. This approach shows a new efficient wavelength conversion scheme, and we explore the cascaded OCR to enlarge the span of the wavelength conversion.

We show the flexible dispersion profiles with three controlled ZDWs, $\lambda_{1}<\lambda_{2}<\lambda_{3}$, as shown in Fig. 1(a), by tuning the pitch $\Delta$ and the relative hole size $D=d / \Delta$ in PCFs. $\lambda_{2}$ and $\lambda_{3}$ shift to longer wavelengths as the increase of $D$. Three anomalous GVD regions separated normal GVD are formed. Fig. 1(b) shows the predicted PM wavelength versus soliton wavelength when the input pulse has a full width at half-maximum (FWHM) of 50 fs and the soliton number $\mathrm{N}=1$. The soliton PM condition is satisfied at a so-called critical point (CP), where two radiation bands are degenerate. Two different CPs are chosen to make soliton spectral tunneling in two-segment PCFs.
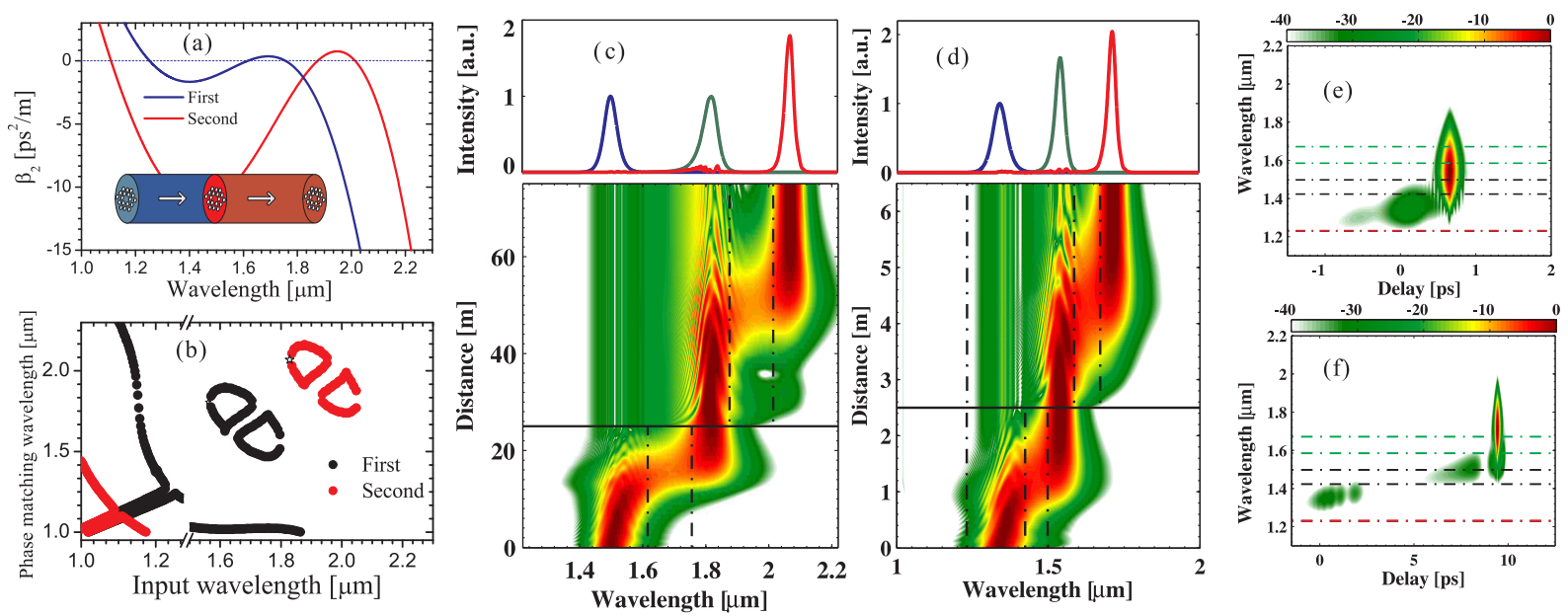

Fig. 1 (a) Chromatic dispersion profiles for the first and second PCFs with $\Delta=2.258 \mu \mathrm{m}, D=0.275$ and $\Delta=2.26 \mu \mathrm{m}, D=0.305$ (inset is the schematic of two-segmented PCFs). (b) Phase matching curves between the soliton and the phase-matching wavelengths, $\star$ indicate the critical point (CP). (c) Spectral evolution of $50 \mathrm{fs}(\mathrm{FWHM})$ input soliton $(\mathrm{N}=1)$ at the pump wavelength of $1.50 \mu \mathrm{m}$ in two-segmented PCFs.

(d) Spectral evolution of $35 \mathrm{fs}(\mathrm{FWHM})$ input soliton $(\mathrm{N}=1)$ at the pump wavelength of $1.34 \mu \mathrm{m}$ in two-segmented TCFs. (e) and (f) Spectrograms of the output pulses in the first and second TCFs.

Fig. 1(c) shows that the cascaded SST leads to an output pulse at the important wavelength range around $2 \mu \mathrm{m}$ from the input pulse at $1.50 \mu \mathrm{m}$ by employing two-segment PCFs. The radiation wavelength at the CP soliton $(1.56 \mu \mathrm{m})$ in the first PCfs is $1.83 \mu \mathrm{m}$ and red-shifts to the second $\mathrm{CP}$, after second tunneling the second radiation wavelength is $2.068 \mu \mathrm{m}$. Large wavelength conversion span over $500 \mathrm{~nm}$ are achieved. Nonlinear soliton matching and mode field matching between two PCFs will be discussed. Fig. 1(d) shows that cascaded SST can also be realized by using two-segment three-cladding fibers (TCF), where the total fiber length is only $6.5 \mathrm{~m}$, which makes experimental observation possible. Note that if proper fiber dimension parameters are selected, we can engineer the output wavelength in near-IR by using well-developed Yb-doped and Er-doped fiber lasers. The above mentioned cascaded SST process is confirmed by using time-frequency cross-correlation frequency-resolved optical gating (XFROG) traces, as shown in Fig. 1 (e) and (f). The output pulses from the first and second fiber remain soliton states.

\section{References}

[1] J. M. Dudley, G. Genty, and S. Coen, "Supercontinuum generation in photonic crystal fiber," Rev. Mod. Phys. 78, 1135-1184 (2006)

[2] B. Kibler, P.-A. Lacourt, F. Courvoisier and J.M. Dudley, "Soliton spectral tunnelling in photonic crystal fibre with sub-wavelength core defect," Electron. Lett. 43, 967-968 (2007). 